\title{
On the Scalability of Uncoordinated Multiple Access for the Internet of Things
}

\author{
Giovanni Chisci, Student Member, IEEE, Hesham ElSawy, Senior Member, IEEE, \\ Andrea Conti, Senior Member, IEEE, Mohamed-Slim Alouini, Fellow, IEEE, and Moe Z. Win, Fellow, IEEE
}

\begin{abstract}
The Internet of things (IoT) will entail massive number of wireless connections with sporadic traffic patterns. To support the IoT traffic, several technologies are evolving to support low power wide area (LPWA) wireless communications. However, LPWA networks rely on variations of uncoordinated spectrum access, either for data transmissions or scheduling requests, thus imposing a scalability problem to the IoT. This paper presents a novel spatiotemporal model to study the scalability of the ALOHA medium access. In particular, the developed mathematical model relies on stochastic geometry and queueing theory to account for spatial and temporal attributes of the IoT. To this end, the scalability of the ALOHA is characterized by the percentile of IoT devices that can be served while keeping their queues stable. The results highlight the scalability problem of ALOHA and quantify the extend to which ALOHA can support in terms of number of devices, traffic requirement, and transmission rate.
\end{abstract}

Index Terms-Internet of things, Poisson point process, wireless networks, medium access control, communication delay.

\section{INTRODUCTION}

The Internet of things (IoT) bridges our physical world to the Internet, leading to a new era of connectivity, networking, monitoring, surveillance, automation, and control. It is foreseen that the IoT will emerge in different sectors (e.g., smart cities, public safety, health-care, vehicular networks, commerce etc.) that will flourish several industries and enhance numerous aspects in our world [1], [2]. Each of the IoT sectors will entail massively many connected things including user equipments, sensors, machines, and vehicles, which are required to communicate and/or connect to the Internet with certain quality of service (QoS) constraints. Consequently, developing a scalable wireless networking platform that is able to accommodate a massive number of devices with diverse QoS constraints is mandatory to unlock the potentials and reap the benefits of IoT.

Spectrum access and reuse are among the fundamental challenges for IoT networking. The massive number of devices,

G. Chisci and A. Conti are with the Department of Engineering (ENDIF), University of Ferrara, Ferrara, Italy (e-mail: giovanni.chisci@unife.it, a.conti@ieee.org). H. ElSawy and M.-S. Alouini are with the Computer, Electrical and Mathematical Science and Engineering Division (CEMSE), King Abdullah University of Science and Technology (KAUST), Thuwal, Saudi Arabia, (e-mail \{hesham.elsawy, slim.alouini\}@kaust.edu.sa). M. Z. Win is with the Laboratory of Information and Decision Systems (LIDS), Massachusetts Institute of Technology (MIT), Cambridge, Massachusetts, USA (e-mail: moewin@mit.edu).

This research was supported, in part, by the " $5 \times 1000 "$ Young Researcher Mobility Project, University of Ferrara, Ferrara, Italy, and by the Sensor Research Initiative through the Office of Sponsored Research at the King Abdullah University of Science and Technology, Thuwal, Saudi Arabia. multitude diversity in their types, intrinsic sporadic traffic patterns, wide-spatial existence, and diverse QoS constraints impose several new challenges to wireless communications and networking. To cope with these challenges, there are several evolving large-scale and low-power wireless technologies that are tailored to the IoT characteristics [3], such as LoRa, SigFox, On-Ramp Wireless, and Weightless. The 3GPP LTE standard also provides solutions for accommodating IoT traffic within the cellular networks such as device-to-device communications, machine-to-machine communications, and narrowband IoT (NB-IoT) [4]. Each of these technologies provides innovative solutions in terms of energy efficiency, spectrum sharing, and traffic prioritization to serve IoT applications. However, uncoordinated random spectrum access is a common ground in all of these technologies, and represents a major scalability bottleneck [3]. In particular, variations of ALOHA and CSMA are used either to transmit data and/or to request scheduling. Hence, a severe performance degradation due to collisions occurs as the number of devices grows.

To efficiently design and characterize the scalability of the random access process, rigorous mathematical models that capture the essence of IoT networks are required. Conventionally, stand-alone queueing models were used to characterize and design the random access in wireless networks. For instance, the seminal work in [5] triggered a plethora of studies that helped developing carrier sensing random access. However, the stream of work based on [5] considers a singlenode queueing model with a constant collision probability. The work in [6] models the random access process via a network of interacting queues and give more realistic parametrization for the collision probability. However, communication failures due to interference are abstracted via the pessimistic collision model. A step forward to account for the network topology and physical layer attributes (e.g., fading and path-loss) is achieved by merging graph theory and queuing theory in [7], where neighborhood restricted interactions fail to capture the aggregate effect from non-neighbors, which may by significant in IoT due to the massive numbers of devices. To account for the interaction between the massively existing IoT devices in a realistic signal-to-interference-plus-noise ratio (SINR) capture model, stochastic geometry and queueing theory are jointly utilized in [8], [9]. However, [8] focuses on the sufficient and necessary conditions for queue stability and [9] is confined to IoT within cellular networks. Furthermore, the work in [9] utilizes the spatially averaged performance for all coexisting IoT devices, which might not always be an accurate estimation as shown in this paper. It is worth noting that there are notable 
stochastic geometry models that account for detailed physicallayer parameters and network topology [10]-[13]. However, stand-alone stochastic geometry models abstract the temporal attributes such as traffic generation patterns, queueing stability, and packet delay, which are fundamentals in IoT networks.

This paper utilizes stochastic geometry and queueing theory to develop a novel spatiotemporal model for ALOHAbased IoT networks. A basic slotted ALOHA scheme is particularly selected for its low-complexity ${ }^{1}$ and mathematical convenience. More sophisticated random access models are left for future extensions. The developed mathematical model abstracts the IoT network to a network of interacting data queues where interactions are captured via stochastic geometry. To this end, a static scenario is considered, where the node scattering is arbitrary, yet fixed. Thus, the analysis is carried out by the meta distribution of the SINR [15], which is utilized to divide the nodes into quality of service (QoS) classes according to their location dependent performance.

The results show the tradeoffs between the scalability and traffic generation in IoT networks as well as enabling the design of access policies for guaranteed stability for a certain percentile of users. One of the main messages is the existence of an optimal ALOHA probability, which mediates between unnecessary transmission deferral due to a too conservative policy and high transmission failure due to high interference, which is caused by a too aggressive policy.

\section{A. Notation}

Along the paper we use the following notation: random variable $(\mathrm{RV}) \mathrm{v}$, variable $v$, random vector $\mathbf{v}$, vector $\boldsymbol{v}$, random matrix $\mathbf{V}$, matrix $\boldsymbol{V}$, random set $\Omega$, set $\Omega$, probability density function (PDF) $f_{\mathrm{v}}(\cdot)$, cumulative distribution function (CDF) $F_{\mathrm{v}}(\cdot)$, complementary cumulative distribution function (CCDF) $\bar{F}_{\mathrm{v}}(\cdot)$, Laplace transform (LT) $\mathcal{L}_{\mathrm{v}}(\cdot),{ }^{2}$ probability mass function (PMF) $F_{\mathrm{v}}(\cdot)$, complement $\bar{v}=1-v$, Kronecker delta function $\delta(\cdot)$.

\section{SYSTEM MODEL}

For the sake of organized presentation, the spatial and physical layer system model are first presented and then followed by the temporal and MAC layer attributes.

\section{A. Spatial and Physical Layer Parameters}

This paper considers an IoT network in which the devices are scattered in $\mathbb{R}^{2}$ according to a Poisson bipolar network (PBN) [16, Def. 5.8]. That is, the potential transmitters belongs to a Poisson point process (PPP) $\Pi=\left\{\mathbf{y}_{j} \in \mathbb{R}^{2}\right\}, j=$ $1,2, \ldots$, with intensity $\lambda$ and each transmitter communicates with a dedicated receiver located at a fixed distance $R$ in uniform random orientation. A realization of the PBN is shown in Fig. 1. For simplicity, we utilize an unbounded path loss model $\ell(r)=r^{-2 \eta}$, where $r$ is the propagation distance and $\eta$

\footnotetext{
${ }^{1}$ IoT networks (e.g., SigFox, LoRa, and Weightless) and LTE NB-IoT rely on ALOHA random access [3], [14].

${ }^{2}$ From now till the rest of the paper we will abuse the terminology "LT of the RV v" for the LT of its PDF.
}

is the amplitude exponent. We consider a Rayleigh fading with mean power $\mu$, i.e., $F_{\mathrm{h}}(h)=1-e^{-\mu h}$ and $\mathcal{L}_{\mathrm{h}}(s)=\frac{1}{\mu s+1}$. From now on we will assume $\mu=1$ for simplicity of treatise. All terminals transmit with a fixed power $P_{\mathrm{T}}$ and universal frequency reuse is employed.

\section{B. Temporal Parameters and Queuing Model}

We consider a discrete-time system with slot duration of $T_{\mathrm{s}}$ seconds. Each transmitter has a buffer that stores data received from higher layers. An independent and identically distributed geometric traffic generation model, with per-slot probability of $0<a<1$, is assumed at each buffer. At each time slot, transmitters with non-empty buffers employ a slotted ALOHA protocol with transmission probability $p_{\mathrm{A}}$ to access the channel and transmit one packet of size $T_{\mathrm{s}} R_{\mathrm{t}}$ from their buffers, where $R_{\mathrm{t}}=W \log (1+\theta) \quad$ [bits/second] is the fixed transmission rate employed by transmitters upon a channel access. Hence, the packet is successfully decoded at the intended receiver if the SINR exceeds $\theta$. Packets are transmitted according to first in-first out (FIFO) rule and only successfully transmitted packets are dropped from the transmitters' buffers. Otherwise, the packet is kept in the buffer until successful transmission.

\section{Methodology}

The operation of the aforementioned network involves intricate and interdependent interactions among the spatially coexisting devices. Since only devices with non-empty queues contend for spectrum access, the aggregate interference and SINR statistics at a given receiver depend on the steadystate probabilities of the queues. Concurrently, steady-state probabilities of the queues depend on the departure rates of packets, which are functions of the aggregate interference and SINR statistics at the receivers. To analyze networks with the aforementioned intricate interactions among devices, we use the following approach; i) use stochastic geometry to characterize the SINR as a function of the activity factors of the devices; ii) use queueing theory to characterize the steadystate probabilities of the queues and, hence, activity factors of devices as functions of the SINR; and iii) find the solution for the system of equations generated by steps (i) and (ii).

\section{SINR CHARACTERIZATION}

The first step to analyze the SINR is to mark the point process of the transmitters by their activity parameters as $\Pi=\left\{\mathbf{y}_{j}, \mathrm{a}_{j}, \mathrm{q}_{j}\right\}$, where $\mathrm{a}_{j}, \forall j$, are independent and identically distributed (i.i.d.) Bernoulli marks for ALOHA channel access, and $\mathrm{q}_{j}, \forall j$, are random integers that represent the queue states (i.e., number of packets in the device queue). The Bernoulli marks $a_{j}$ are parameterized with the channel access probability $p_{\mathrm{A}}$. The distribution of the queue states $\mathrm{q}_{j}$ is to be determined in Section IV.

In the static network, it is assumed that the network constitutes a fixed, but arbitrary, realization of the PBN. For an arbitrary transmitting ${ }^{3}$ test link in $\Pi$, the transmission success probability is defined as:

\footnotetext{
${ }^{3}$ Transmission requires activity (i.e., non-empty queue) and ALOHA channel access.
} 


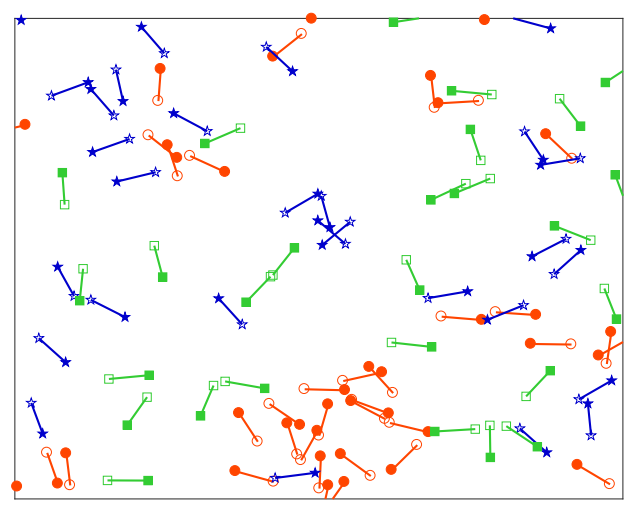

Fig. 1: A realization of PBN with $N=3$ QoS classes, where the filled (empty) shapes represent transmitters (receivers). The green squares, blue pentagrams, and red circles represent the high $\left(\mathrm{p}_{\mathrm{s}}>0.78\right)$, medium $\left(0.65<\mathrm{p}_{\mathrm{s}}<0.78\right)$, and low $\left(\mathrm{p}_{\mathrm{s}}<\right.$ $0.65)$ QoS links, respectively.

$$
\mathrm{p}_{\mathrm{s}}=\mathbb{P}\left\{\frac{P_{\mathrm{T}} \mathrm{h}_{\circ} R^{-2 \eta}}{\sum_{j: \mathbf{y}_{j} \in \Pi_{\mathrm{I}}} P_{\mathrm{T}} \mathrm{h}_{j}\left\|\mathbf{y}_{j}\right\|^{-2 \eta} \mathrm{a}_{j} \mathbb{1}_{\left\{\mathrm{a}_{j}>0\right\}}+\sigma^{2}}>\theta \mid \Pi\right\},
$$

where $h_{\circ}$ is the intended channel gain, $\Pi_{I}=\Pi \backslash y_{\circ}$ is the set of transmitters excluding the intended test transmitter. Note that not all the transmitters in $\Pi_{I}$ are interfering at the test receiver. The activity of interferers due to their buffers states is captured by $\mathbb{1}_{\left\{q_{j}>0\right\}}$, and the ALOHA channel access effect is captured by $\mathrm{a}_{j}$.

Since the network realization is static, the transmission success probabilities of the PBN links are location dependent. As shown in Fig. 1, links that exist in a condensed region in $\mathbb{R}^{2}$ would have poorer transmission success probabilities than links that exist in a sparse region. The recently developed theory of the meta distribution of the SINR for the PBN [15] models such discrepancies in the devices performance. Particularly, [15] shows that the conditional distribution of the transmission success probability $\mathrm{p}_{\mathrm{s}}$ across the links in a static PBN can be accurately approximated by the Beta distribution

$$
\begin{aligned}
f_{\mathrm{p}_{\mathrm{s}}}(\phi) & =\frac{\phi^{\frac{M_{1}(\alpha+1)-1}{1-M_{1}}}(1-\phi)^{\alpha-1}}{B\left(\frac{M_{1} \alpha}{1-M_{1}}, \alpha\right)} \\
\alpha & =\frac{\left(M_{1}-M_{2}\right)\left(1-M_{1}\right)}{M_{2}-M_{1}^{2}}
\end{aligned}
$$

where $B(\cdot, \cdot)$ is the complete Beta function and $M_{1}$ and $M_{2}$ are the first and second order moment of $p_{s}$, respectively, i.e.,

$$
\begin{aligned}
M_{1} & \triangleq \mathbb{E}^{! \circ}\left\{\mathrm{p}_{\mathrm{s}}\right\}=\exp \left\{-C \theta^{\frac{1}{\eta}} R^{2} p_{\mathrm{A}} p_{\mathrm{Q}}-\hat{\theta} \sigma^{2}\right\} \\
M_{2} & \triangleq \mathbb{E}^{! \circ}\left\{\mathrm{p}_{\mathrm{s}}^{2}\right\} \\
& =\exp \left\{-C \theta^{\frac{1}{\eta}} R^{2} p_{\mathrm{A}} p_{\mathrm{Q}}\left(2-\left(1-\frac{1}{\eta}\right) p_{\mathrm{A}} p_{\mathrm{Q}}\right)-2 \hat{\theta} \sigma^{2}\right\}
\end{aligned}
$$

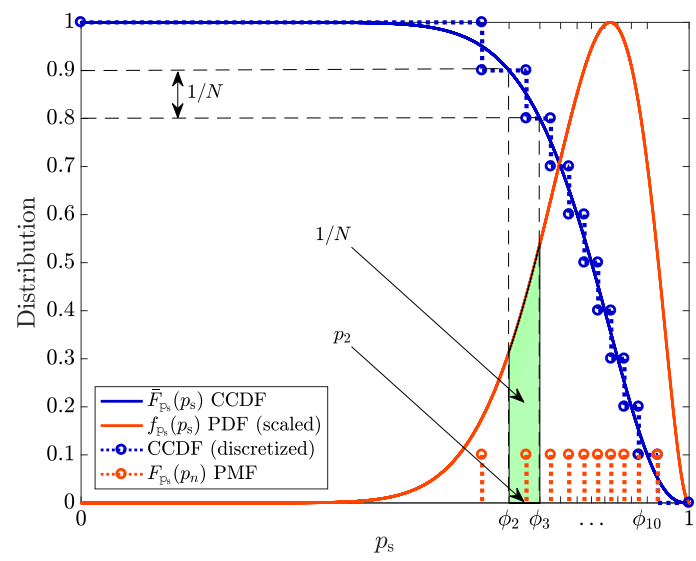

Fig. 2: Distribution of the continuous conditional success probability and its discretization by importance sampling $(N=10)$.

where $p_{\mathrm{Q}}=\mathbb{E}^{! \circ}\left\{\mathbb{1}_{\left\{\mathbf{q}_{j}>0\right\}}\right\}=\mathbb{P}^{! \circ}\left\{\mathbf{q}_{j}>0\right\}$ is the average probability of having a non-empty queue, $\hat{\theta}=\theta R^{2 \eta} / P_{\mathrm{T}}$, and $C=\lambda \pi \Gamma\left(1-\frac{1}{\eta}\right) \Gamma\left(1+\frac{1}{\eta}\right)$.

If each distinct realization of $p_{\mathrm{s}}$ is considered as a QoS class, then infinite number of classes are required to classify the devices. For tractability, we discretize $\mathrm{p}_{\mathrm{s}} \in\left\{p_{1}, p_{2}, \ldots, p_{N}\right\}$ to $N$ equiprobable success probability classes, where the number of classes is selected to achieve a sufficiently accurate mathematical model. Fig. 1 shows a realization of the PBN with $N=3$ QoS classes, defined as $\mathrm{p}_{\mathrm{s}} \in\left\{p_{1}=0.56, p_{2}=\right.$ $\left.0.71, p_{3}=0.84\right\}$, that have equivalent percentiles (i.e. $1 / 3$ each) of users. The network partitioning process by the discretization of the PDF in (2) is described in Algorithm 1 and visualized in Fig. 2. In particular, step 2 divides the $p_{\mathrm{s}}$ axis in $N$ intervals with different length corresponding to the $1 / N$ percentiles of users and step 3 computes per-class success probability by taking the median value over each interval. As shown in Fig. 2, Algorithm 1 provides an importance discretization scheme that puts more samples at locations of $f_{\mathrm{ps}_{\mathrm{s}}}(\phi)$ with higher variability. Moreover, step 3 minimizes the average error of $\operatorname{argmin}_{n}\left\|p_{n}-\mathrm{p}_{\mathrm{s}}\right\|$.

\footnotetext{
Algorithm 1 Success probability mark discretization

Require: $M_{1}, M_{2}, \mathrm{~N}, \phi_{1}=0, \phi_{N+1}=1$.

Ensure: A set of success probability values $p_{n}$ with PMF $F_{\mathrm{p}_{\mathrm{s}}}\left(p_{n}\right)=1 / N$ for all $n=1,2, \ldots, N$.

1: for $\mathrm{n}=1,2, \ldots, \mathrm{N}$ do

2: $\quad$ Retrieve the values of $\phi_{n}$ and $\phi_{n+1}$ such that (s.t.)

$$
F_{\mathrm{p}_{\mathrm{s}}}\left(\phi_{n}\right)-F_{\mathrm{p}_{\mathrm{s}}}\left(\phi_{n+1}\right)=\int_{\phi_{n}}^{\phi_{n+1}} f_{\mathrm{p}_{\mathrm{s}}}(\phi) d \phi=\frac{1}{N}
$$

3: $\quad$ Compute $p_{n}$ s.t. $\int_{\phi_{n}}^{p_{n}} f_{\mathbf{p}_{\mathrm{s}}}(\phi) d \phi=\int_{p_{n}}^{\phi_{n+1}} f_{\mathrm{p}_{\mathrm{s}}}(\phi) d \phi$ and assign $F_{\mathrm{p}_{\mathrm{s}}}\left(p_{n}\right)=\frac{1}{N}$ 


\section{QUEUEING ANALYSIS}

From the temporal perspective, each link of the PBN is abstracted to a queue that tracks the number of packets in the transmission buffer. The service rate of such a queue is determined by the SINR statistics (i.e., transmission success probability) at the intended receiver. This section presents the queueing analysis for the static PBNs, by assuming to know the queue service rates.

Since the transmission success probability depends on the QoS class, an explicit queueing analysis for each class is necessary. It is assumed that each link within the $n^{\text {th }}$ QoS class experiences independent transmission success probability, with mean $p_{n}$, across time slots. Such an assumption, which is validated in Section VI, ignores the temporal correlation of the SINR across time to alleviate queue memory complications. By exploiting the temporal independence of success probabilities and the fact that only one packet can arrive and/or depart from the queue in each slot, each link within the $n^{\text {th }}$ QoS class is abstracted to a $\mathrm{Geo} / \mathrm{Geo} / 1$ queueing system with infinite buffer and the following transition matrix:

$$
\boldsymbol{P}_{n}=\left[\begin{array}{cccc}
\bar{a} & a & & \\
\bar{a} b_{n} & a b_{n}+\bar{a} \bar{b}_{n} & a \bar{b}_{n} & \\
& \bar{a} b_{n} & a b_{n}+\bar{a}_{n} & a \bar{b}_{n} \\
& \ddots & \ddots & \ddots
\end{array}\right]
$$

where $b_{n}=p_{\mathrm{A}} p_{n}$ is the probability that a packet departs from the queue (i.e., successfully received at the intended receiver) of a transmitter that belongs to the $n^{\text {th }}$ QoS class. Note that the departure requires both ALOHA channel access and transmission success. On the other hand, the packet does not depart with probability $\bar{b}_{n}=\bar{p}_{A}+p_{\mathrm{A}} \bar{p}_{n}$ which is a consequence of either ALOHA channel access deferral or transmission failure upon channel access. With simple manipulations, it can be shown that $\bar{b}_{n}=1-p_{\mathrm{A}} p_{n}$.

The steady-state distribution of a queue with transition matrix $\boldsymbol{P}_{n}$ is obtained by solving the following system of equations

$$
\begin{aligned}
\boldsymbol{x}_{n} \boldsymbol{P}_{n} & =\boldsymbol{x}_{n} \\
\boldsymbol{x}_{n} \mathbf{1} & =1
\end{aligned}
$$

where $\boldsymbol{x}_{n}=\left[x_{0, n}, x_{1, n}, x_{2, n}, \ldots\right]$ is the steady-state distribution of the number of packets in the buffer for a transmitter that belongs to the $n^{\text {th }}$ QoS class, and $\mathbf{1}$ is a vector of ones with the appropriate length. The vector $\boldsymbol{x}_{n}$ in (6) gives the PMF of the queue state random variable (i.e., $\mathbb{P}^{! \circ}\left\{\mathbf{q}_{j}=i \mid \mathbf{y}_{j} \in\right.$ $n^{\text {th }}$ class $\}=x_{i, n}$ ) for the $j^{\text {th }}$ transmitter of the $n^{\text {th }}$ QoS class. Following [17], the queue is stable if and only if (i.f.f.)

$$
\frac{p_{\mathrm{A}} p_{n}}{a}<1
$$

In case of stability, the system in (6) has the following closed form steady-state solution

$$
\begin{aligned}
x_{0, n} & =\frac{p_{\mathrm{A}} p_{n}-a}{p_{\mathrm{A}} p_{n}} \\
x_{l, n} & =\rho_{n}^{l} \frac{x_{0, n}}{p_{\mathrm{A}} p_{n}} \\
\rho_{n} & =\frac{a\left(1-p_{\mathrm{A}} p_{n}\right)}{\bar{a} p_{\mathrm{A}} p_{n}} .
\end{aligned}
$$

After solving the system of equations in (8) for all QoS classes, the probability that the buffer of a generic transmitter is nonempty is estimated by

$$
p_{\mathrm{Q}}=\mathbb{P}^{! \circ}\left\{\mathrm{q}_{j}>0\right\} \simeq \frac{1}{N} \sum_{n=1}^{N}\left(1-x_{0, n}\right)
$$

which is required in (3) to characterize the distribution of $\mathrm{p}_{\mathrm{s}}$ given in (2). Note that the approximation is due to the discretization of the meta distribution (see Algorithm 1).

Due to the discrepancies among the departure rates of the QoS classes, some devices may not be stable. Using the stability definition in (7), the percentile of stable users (PSU) is defined as

$$
\gamma_{\mathrm{su}} \triangleq \mathbb{P}\left\{p_{\mathrm{A}} \mathrm{p}_{\mathrm{s}}>a\right\}=\bar{F}_{\mathrm{p}_{\mathrm{s}}}\left(\frac{a}{p_{\mathrm{A}}}\right)
$$

which is equal to the theoretical probability of stability at the typical point $\bar{F}_{\mathrm{p}_{\mathrm{s}}}\left(\frac{a}{p_{\mathrm{A}}}\right)$ (i.e., the CCDF related to the Beta distribution in (2) and (3)). For a sufficiently high $N$ and thanks to the ergodicity of the PBN $\gamma_{\mathrm{su}}$ is estimated by

$$
\widehat{\gamma}_{\mathrm{su}}=\frac{1}{N} \sum_{n=1}^{N} \mathbb{1}_{\left\{p_{\mathrm{A}} p_{n}>a\right\}} .
$$

Thus, we introduce the network $\gamma$-stability, which is achieved if the percentile of stable users is greater than a target percentile $\gamma$, i.e., $\gamma_{\mathrm{su}}>\gamma$.

\section{Overall Network Performance}

This section provides the joint solution of the stochastic geometry and queueing theory problems presented in Section III and Section IV, respectively. However, before delving into the solution details, it is important to explicitly state all of the involved approximations.

Approximation 1: The spatial correlation among the devices of the same class is ignored.

Approximation 2: The devices are assumed to be active independently with probability $p_{\mathrm{Q}}$ (average activity probability over classes) and the transmission successes are independent over different time slots with class dependent probability $p_{n}$. We validate the approximations in Section VI.

Note from (7), (8), and (9) that the stability condition, the steady-state solution, and the probability of non-empty queues in a static PBN are functions of $p_{n}$, which are computed through Algorithm 1. Concurrently, $p_{n}$ requires $M_{1}$ and $M_{2}$ which are functions of $p_{\mathrm{Q}}$ which is obtained by (9) through the queueing steady-state solutions of every class. Consequently, the system of equations given in (2), (3), (8), and Algorithm 1 should be solved simultaneously as shown in Algorithm 2.

It is worth noting that while the de facto queue stability should be determined through Algorithm 2, the necessary and sufficient conditions for the network $\gamma$-stability can be determined, respectively, as

$$
\begin{aligned}
& \gamma_{\mathrm{su}}\left(p_{\mathrm{Q}}=a\right)=\bar{F}_{\mathrm{p}_{\mathrm{s}}}\left(\frac{a}{p_{\mathrm{A}}} \mid p_{\mathrm{Q}}=a\right)>\gamma \\
& \gamma_{\mathrm{su}}\left(p_{\mathrm{Q}}=1\right)=\bar{F}_{\mathrm{p}_{\mathrm{s}}}\left(\frac{a}{p_{\mathrm{A}}} \mid p_{\mathrm{Q}}=1\right)>\gamma
\end{aligned}
$$

which can be evaluated after computing $M_{1}$ and $M_{2}$ by (3). 


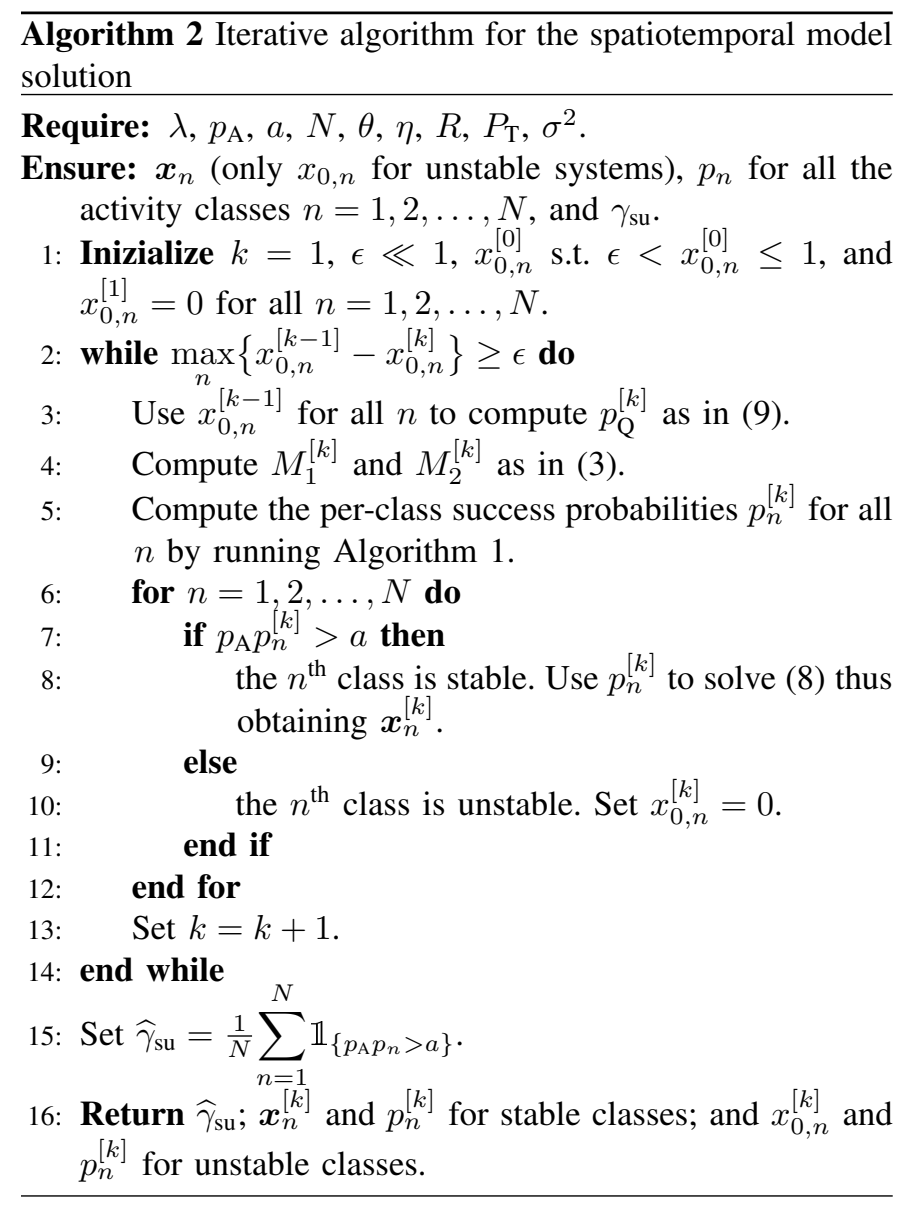

\section{Simulation AND Numerical Results}

We first validate the theoretical results via independent Monte Carlo simulations. The PBN is scattered once in a 10 $\times 10 \mathrm{~km}^{2}$ area. However, the fading realization changes at each time slot. In the initial state of the simulation, all buffers are assumed empty. At each time slot of the simulation, the following happens; 1) packets are generated in the buffers according to a Bernoulli random viable with probability $a$; 2) only devices with non-empty buffers contend for spectrum access via ALOHA; 3) the SINR is computed for devices that accessed the channel using a wrap-around network model to avoid boundary effects; and 4) the packets depart from the buffers of the devices that experience an SINR greater than the required threshold $\theta$. The procedure is repeated 10,000 times in which the statistics are only recorded after the convergence to the steady-state, which is detected by the stabilization of $p_{\mathrm{Q}}$. The meta distribution of the SINR is then evaluated across all devices to plot the simulation curves shown in Fig 3 .

Fig 3 shows a perfect match between the meta distributions obtained via simulations and those obtained analytically via Algorithm 2 for $N=10$, which validates the developed mathematical model. Note that the close match shown in Fig 3 is due to the correct estimation of the moments. For instance, the moments for the second scenario are $M_{1}=0.1679$, $M_{1}^{\text {sim }}=0.1638, M_{2}=0.0445, M_{2}^{\text {sim }}=0.0429$, thus showing errors smaller than $4 \%$ and $3 \%$, respectively. Fig. 3 also shows the inaccuracy of Algorithm 2 if an insufficient number of QoS

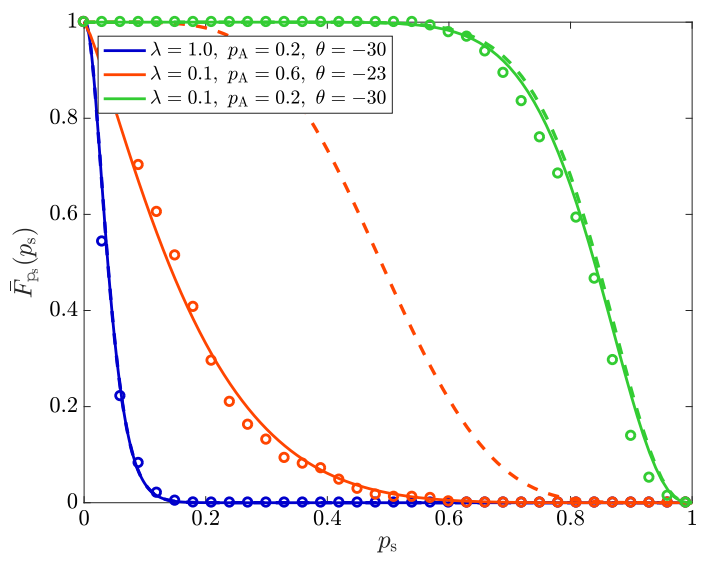

Fig. 3: CCDFs of $p_{s}$ in different network scenarios. Theoretical values are shown for $N=10$ (solid lines) and $N=1$ (dashed lines) along with simulated values (dotted lines) for $\eta=2, R=10[\mathrm{~m}], \sigma^{2}=-90[\mathrm{dBm}], P_{\mathrm{T}}=-30[\mathrm{dBm}]$, $a=0.1$ [packets/slots].

classes is used. This happens because of a wrong estimation of $p_{\mathrm{Q}}$ by (9), which propagates through the iterative algorithm.

Fig. 4 shows the stability regions over three different parameter planes. In each figure, solid lines are $\gamma$-stability frontiers for $\gamma=0.1,0.2, \ldots, 0.9$ while the marked dashed line shows the deterministic stability frontier obtained by the traditional spatially averaged transmission success probability (i.e., by the condition $p_{\mathrm{A}} M_{1}<a$ ). It can be observed in all the three figures that, for a given target stability, our methodology enables the determination of the stability frontier with more accuracy than classical methodologies based on spatial averaging.

Moreover, Fig. 4a shows on the $(\theta, \lambda)$-plane that a higher percentile of users can be ensured to be stable if the node density and SINR threshold are small, i.e., low aggregate interference and small SINR threshold. Fig. $4 \mathrm{~b}$ presents on the $\left(p_{\mathrm{A}}, \lambda\right)$-plane the fact that neither a too conservative nor a too aggressive access policy ensures stability. Given a $\lambda$, to employ a conservative access policy with low values of $p_{\mathrm{A}}$ misses many transmission opportunities, accumulates packets in buffers, and leads to instability. On the other hand, to employ an aggressive access policy with high values of $p_{\mathrm{A}}$ aggravates the interference, leads to transmission failures due to low SINR, and hence leads to instability. The values of $p_{\mathrm{A}}$ that guarantee stability differ with $\lambda$ and the required percentile of stable devices $\gamma$. For $\lambda$ values smaller than the critical value $\left(\lambda<\lambda^{\mathrm{c}}=0.167\right)$ the the $90 \%$-stability would not hold only due to packet accumulation because of the sparse node scattering. Fig. $4 \mathrm{c}$ illustrate on the $\left(p_{\mathrm{A}}, a\right)$-plane the feasibility condition for stability. Intuitively, when the interference is negligible and the success upon a transmission is almost certain, the stability condition becomes $p_{\mathrm{A}}>a$, which explain the absence of stable points above the $p_{\mathrm{A}}=a$ line. Note that given a $\gamma$, for increasing $a$ the minimum $p_{\mathrm{A}}$ ensuring $\gamma$-stability diverges increasingly from $p_{\mathrm{A}}=a$. This happens because $p_{\mathrm{A}}$ has to compensate the occurrence of failures due to high queue occupation and, hence, interference. 


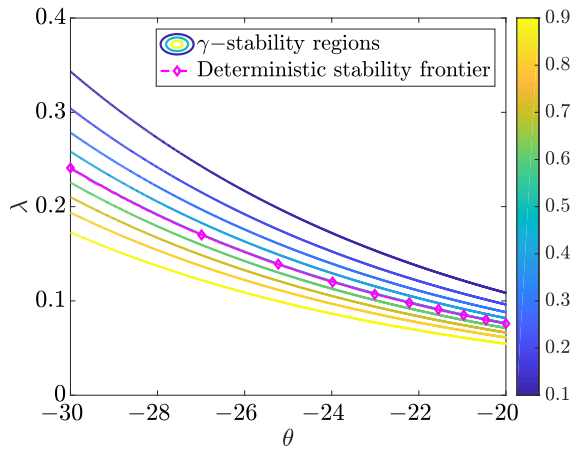

(a) $(\theta, \lambda)$-plane.

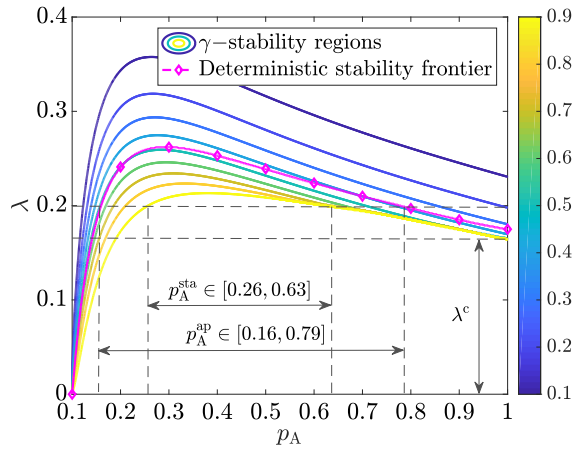

(b) $\left(p_{\mathrm{A}}, \lambda\right)$-plane.

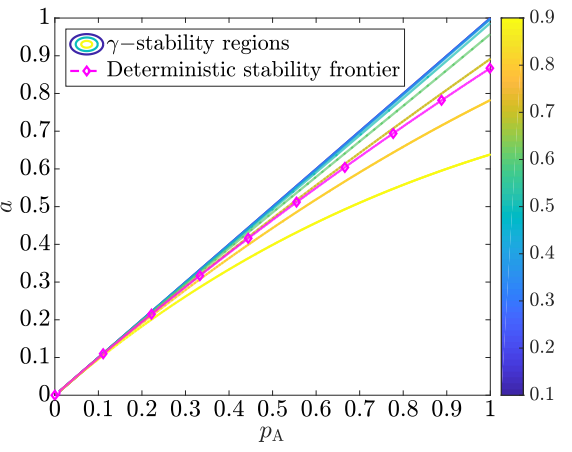

(c) $\left(p_{\mathrm{A}}, a\right)$-plane.

Fig. 4: Stability regions over three different parameter planes. We show $\gamma$-stablity regions for $N=10$ (colorplot) and the deterministic stability frontier for the average performance (marked curve). If not stated differently $\lambda=0.01\left[\right.$ nodes $\left./ \mathrm{m}^{2}\right]$, $\eta=2, R=10[\mathrm{~m}], \theta=-30[\mathrm{~dB}], \sigma^{2}=-90[\mathrm{dBm}], P_{\mathrm{T}}=-30[\mathrm{dBm}], a=0.1$ [packets/slots], $p_{\mathrm{A}}=0.2$ [attempts/slots].

\section{A. Design Insights}

From the design perspective, it is better to select the $p_{\mathrm{A}}$ that maximizes the product $p_{\mathrm{A}} p_{\mathrm{s}}$. In facts, when stability holds [9], QoS metrics such as the number of attempts before a success, the queuing length, and the waiting time in queue are monotonically decreasing functions of the departure rate $p_{\mathrm{A}} p_{\mathrm{s}}$. A proposal for the object to be maximized by exhaustive search among the parameter sets holding stability is $p_{\mathrm{A}} M_{1}$.

We conclude by presenting a case study of the design of a network with $\lambda=0.2, \theta=-30[\mathrm{~dB}], P_{\mathrm{T}}=-30[\mathrm{dBm}]$, $\sigma^{2}=-90[\mathrm{dBm}], a=0.1, \eta=2, R=10[\mathrm{~m}]$ and $N=10$. The considered setting is in agreement with the LoRa standard, and corresponds to an ad-hoc network scenario with mediumlow node density (10 nodes on a $10 \times 10\left[\mathrm{~m}^{2}\right]$ squared surface), low power consumption, and medium-range communications. To guarantee the stability for the $90 \%$ of the users $p_{\mathrm{A}}^{\text {sta }} \in$ $[0.26,0.63]$ must be adopted. If one consider the averaged performance, the network is stable for $p_{\mathrm{A}}^{\text {ap }} \in[0.16,0.79]$. The range of $p_{\mathrm{A}}^{\text {sta }}$ is more narrow, because it is intuitively more difficult to guarantee stability for the $90 \%$ of the network than guessing stability "on average".

\section{CONCLUSION}

This paper develops a novel spatiotemporal model for ALOHA-based IoT networks. The developed model enables the abstraction of the IoT as a network of interacting queues that is analyzed via stochastic geometry and queuing theory. The tradeoffs and relations between the network scale and traffic generation are derived. In particular, we show that the channel access influence on network stability is twofold: a too conservative access policy leads to instability due to unnecessary transmission deferrals, while a too aggressive one leads to instability due to the high interference and transmission failures. The network design is addressed such as the stability is guaranteed for a desired percentile of users. Results obtained using our methodology with respect to classical approaches based on the average performance enable a deeper understanding of the fundamental limits of the scalability and traffic generation in IoT networks.

\section{REFERENCES}

[1] A. Al-Fuqaha, M. Guizani, M. Mohammadi, M. Aledhari, and M. Ayyash, "Internet of things: A survey on enabling technologies, protocols, and applications," IEEE Commun. Surveys Tuts., vol. 17, no. 4, pp. 2347-2376, Fourthquarter 2015.

[2] D. S. Nunes, P. Zhang, and J. S. Silva, "A survey on human-in-the-loop applications towards an Internet of all," IEEE Commun. Surveys Tuts., vol. 17, no. 2, pp. 944-965, Secondquarter 2015.

[3] A. Laya, C. Kalalas, F. Vazquez-Gallego, L. Alonso, and J. AlonsoZarate, "Goodbye, ALOHA!" IEEE Access, vol. 4, pp. 2029-2044, 2016.

[4] A. Bader, H. ElSawy, M. Gharbieh, M. S. Alouini, A. Adinoyi, and F. Alshaalan, "First mile challenges for large-scale IoT," IEEE Commun. Mag., vol. 55, no. 3, pp. 138-144, March 2017.

[5] G. Bianchi, "Performance analysis of the IEEE 802.11 distributed coordination function," IEEE J. Sel. Areas Commun., vol. 18, no. 3, pp. 535-547, March 2000.

[6] W. Luo and A. Ephremides, "Stability of $\mathrm{N}$ interacting queues in random-access systems," IEEE Trans. Inf. Theory, vol. 45, no. 5, pp. 1579-1587, Jul. 1999.

[7] G. D. Celik and E. Modiano, "Scheduling in networks with time-varying channels and reconfiguration delay," IEEE/ACM Trans. Netw., vol. 23, no. 1, pp. 99-113, 2015.

[8] Y. Zhong, M. Haenggi, T. Q. S. Quek, and W. Zhang, "On the stability of static Poisson networks under random access," IEEE Trans. Commun., vol. 64, no. 7, pp. 2985-2998, Jul. 2016.

[9] M. Gharbieh, H. ElSawy, A. Bader, and M. Alouini, "Spatiotemporal stochastic modeling of IoT enabled cellular networks: Scalability and stability analysis," IEEE Trans. Commun., no. 99, 2017.

[10] M. Z. Win, P. C. Pinto, and L. A. Shepp, "A mathematical theory of network interference and its applications," Proc. IEEE, vol. 97, no. 2, pp. 205-230, Feb. 2009, special issue on Ultra-Wide Bandwidth (UWB) Technology \& Emerging Applications.

[11] A. Rabbachin, A. Conti, and M. Z. Win, "Wireless network intrinsic secrecy," IEEE/ACM Trans. Netw., vol. 23, no. 1, pp. 56 - 69, Feb. 2015.

[12] M. Haenggi, J. G. Andrews, F. Baccelli, O. Dousse, and M. Franceschetti, "Stochastic geometry and random graphs for the analysis and design of wireless networks," IEEE J. Sel. Areas Commun., vol. 27, no. 7, pp. 1029-1046, Sep. 2009.

[13] H. ElSawy, A. S. Salem, M.-S. Alouini, and M. Z. Win, "Modeling and analysis of cellular networks using stochastic geometry: A tutorial," IEEE Commun. Surveys Tuts., vol. 19, no. 1, pp. 167-203, Firstquarter 2017.

[14] L. Vangelista, A. Zanella, and M. Zorzi, "Long-range IoT technologies: The dawn of LoRa?" in Future Access Enablers of Ubiquitous and Intelligent Infrastructures. Springer, 2015, pp. 51-58.

[15] M. Haenggi, "The meta distribution of the SIR in Poisson bipolar and cellular networks," IEEE Trans. Wireless Commun., vol. 15, no. 4, pp. 2577-2589, Apr. 2016.

[16] - Stochastic Geometry for Wireless Networks. Cambridge, UK: Cambridge University Press, 2013.

[17] A. S. Alfa, Applied Discrete-Time Queues, 2nd ed. Springer, 2016. 